\title{
The role of nonolfactory context cues in odor identification
}

\author{
RICHARD G. DAVIS \\ Veterans Administration Medical Center, Lexington, Kentucky 40507
}

\begin{abstract}
Odors were identified by 141 persons before and after a nonolfactory cue was presented. The context cue was a color concept, either relevant or irrelevant to the odor identity. The color concept was presented as a word or as the color itself. The familiarity of each odor was judged prior to each identification. The relevant color concepts facilitated identification to a slight, but reliable, degree. Irrelevant color concepts evoked an increase in wrong identifications. Relevant cues increased correct second identifications and increased them to an even greater degree if the odors were judged to be highly familiar.
\end{abstract}

When asked to identify odors in an experimental setting, the average performance for naive persons is low: $50 \%$ correct identifications of a list of 36 odors (Lawless \& Engen, 1977, p. 56), 48\% of 32 (Desor \& Beauchamp, 1974, p. 553), and $45 \%$ of 80 (Cain, 1979, p. 467). These investigators used "ecologically valid" odorants to various degrees (i.e., the odorant was an actual sample of the usual source). Therefore, these low identification rates are probably not due to the lack of typical chemical profiles in the odorants being used. Other investigators have used simple odorant mixtures, and they, too, observed an average of $50 \%$ correct identification rates on first encounters with the odorants (Engen \& Pfaffman, 1960; Engen \& Ross, 1973; Mozell, Smith, Smith, Sullivan, \& Swender, 1969). This low rate of identification is puzzling, especially when contrasted with the powerful memory-evoking potential traditionally at attributed to odors.

Perhaps the absence of the nonolfactory context cues that normally accompany an odor experience is a limiting factor in odor identification. Experiments with odor stimuli usually present odorants as disembodied stimuli, without any additional context cues. If context is enriched, then identification rates may markedly improve. Cain (1979) furnished a form of enriched context by providing feedback on the responses given in a multisession study in which the observers attempted to identify the same odors on several occasions. In this situation, Cain was able to observe rates in the $80 \%$ to $90 \%$ range.

The present study was designed to detect the effects of nonolfactory cues on odor identification. The cues were color concepts, which were related to the odor sources in ecologically valid situations. For

This research was supported by the Veterans Administration. Reprint requests should be sent to Richard G. Davis, Research Psychologist, Veterans Administration Medical Center, Lexington, Kentucky 40507. example, the odor of banana was paired with the color concept "yellow." Context cues, which were presented as words, were intended to contact the semantic level at which name identification occurs.

A nonolfactory cue may interact with the olfactory stimulus at an early level of processing, prior to semantic representations. If so, then a nonverbal cue and a verbal cue could affect identification differently. Therefore, the color concepts were also presented as color patches.

Identification failures are known to occur even though the observer is certain that the odor is highly familiar. This phenomenon has been termed the "tip of the nose" (TON) state (Lawless \& Engen, 1977). The TON phenomenon was described as early as 1968 (Tanyolac, 1968), and TON was discussed in identification studies (Cain, 1979). The TON state was studied by Lawless and Engen (1977), who observed that persons in the TON state are able to verbalize responses as to the general class of the odor, the origins of their prior experiences with the odor as to time and place, and the names of similar odors.

The primary focus of this study was on the contribution of a nonolfactory cue to identification success, but, in particular, when the cue is added to the context after a naming failure has occurred. The key trials of interest were those in which an initial failure to identify the odor was followed by a successful identification on the sound "cued" attempt. Trials of this kind, in which the familiarity judgments are also high, are regarded here as TON events.

\section{METHOD}

\footnotetext{
Subjects

The subjects were 141 persons recruited from an introductory psychology course and were fulfilling a course requirement. Of these persons, 50 were male, 91 were female, and 45 declared themselves as users of tobacco or other smoking products. There were 13 male smokers and 32 female smokers. Their ages ranged from 17 to 34 years, with a mean of $18.9(S D=2.4)$ years.
} 


\section{Stimulus Materials}

Odorants were delivered to the subjects in the form of microencapsulated liquids. These microcapsules were held as thin coatings on paper labels applied to pages in books. This microencapsulation technology is the method patented by the Minnesota Mining and Manufacturing Co. (3M) to Matson (1970) and known as Microfragrances (registered 3M trademark). The 3M Company furnished the odorants as coatings on adhesive-backed labels $1.25 \times 5 \mathrm{~cm}$ with a dark brown background mask color. Twenty odors, which had reen studied previously in this laboratory (Davis, 1979), were chosen from those available in the $3 \mathrm{M}$ fragrance library (Table 1). The odorants are considered to be representative of real objects but should not be considered as highfidelity reproductions.

Two arbitrary sequences, and the reverse orders, of the 20 odors were constructed. These four sequences were used to construct stimulus books in which a single odor label appeared on each page.

\section{Judgments}

The subjects reported two judgments about each odor. The familiarity of each odor was estimated and reported as a category assignment from 1 (very unfamiliar) to 9 (extremely familiar). The subjects were then required to identify the odor. Specifically, they were instructed to "report what you think the odor is" and, further, to "identify the source of the fragrance." The identification response was required twice, with the presentation of an additional nonolfactory context cue in between the two identification responses.

\section{The Trial}

On each trial, the subject marked the odor label to release some odorant and inhaled with the label held near the nose. Immediately thereafter, a degree of familiarity was estimated and recorded on the next page in the response booklet. Without delay, the subject decided an identification response, which was entered on the same page in the response booklet. Twenty seconds after smelling the odor, the subject turned the odor-label page to reveal an additional context cue on the reverse side of the page bearing the odor label. After studying the context cue, the subject was required to make a second identification response.

There were five types of trials presented to each subject. These types were based on the kind of context cue presented between the two identification responses. The context cue was a color (e.g., red or blue) presented as a word or as a patch of that color. The colors were chosen to be relevant to the odor that they followed (e.g., yellow followed banana) or irrelevant (e.g., blue followed banana). Table 1 presents the odor/color assignments. The fifth trial type did not present a context cue but merely required a second identification attempt.

\section{Training Trials}

Three initial trials were presented as training trials. The odors used on these trials were not used subsequently in the sequences. They were selected to have relatively lower recognition than the rest of the odors and to present a nonspecific odor impression, for example, pentanoic acid (sweaty socks), musk (a perfume base), and a perfume (an undesignated fragrance not available to the general public). The cues on the three training trials were an irrelevant color, a no-cue trial, and an irrelevant-word trial.

\section{Odor Sequences}

Each subject judged every odor once. There were four of each trial type given to each subject. All 20 odors were used equally often in each trial type. Four arbitrary sequences of odor presentation were used. The permutation of these parameters produced 20 unique stimulus orders, which were used to construct 20 different odor presentation books. Seven persons were tested with each book (an eighth person was inadvertently included with one book), which resulted in the testing of 141 persons.

\section{Identification Scoring}

The identification responses were scored as correct only if they were veridical. Near misses and far misses (in the sense of Cain, 1979) were classified as incorrect. Certain specific exceptions were allowed. For example, the odorant amyl acetate, referred to here by the label banana, sometimes will evoke the response "orange

Table 1

Odor and Color Cue Assignments and Response Measures

\begin{tabular}{|c|c|c|c|c|c|c|}
\hline \multirow[b]{2}{*}{ Odorant } & \multicolumn{2}{|c|}{ Color Concept Context Cue } & \multirow{2}{*}{$\begin{array}{c}\text { Familiarity } \\
\text { Rating }\end{array}$} & \multirow[b]{2}{*}{ TON } & \multicolumn{2}{|c|}{ Initial Identification Rate } \\
\hline & Relevant & Irrelevant & & & Correct & Incorrect \\
\hline Lemon & Yellow & White & 7.8 & 11 & 55 & 29 \\
\hline Coconut & White & Red & 7.2 & 3 & 48 & 34 \\
\hline Chocolate & Brown & Red & 6.0 & 2 & 46 & 23 \\
\hline Cherry & Red & Yellow & 7.5 & 15 & 40 & 43 \\
\hline Smoke & Gray & Green & 6.4 & 3 & 38 & 43 \\
\hline Grass & Green & Yellow & 4.9 & 15 & 33 & 35 \\
\hline Pickle & Green & White & 6.3 & 1 & 33 & 38 \\
\hline Cedar & Green & Yellow & 5.3 & 6 & 31 & 35 \\
\hline Cinnamon & Brown & Green & 5.3 & 6 & 31 & 31 \\
\hline Banana & Yellow & Red & 6.8 & 7 & 26 & 55 \\
\hline Garlic & White & Brown & 6.2 & 1 & 22 & 53 \\
\hline Grape & Purple & Brown & 6.8 & 20 & 21 & 60 \\
\hline Skunk & Black & Brown & 5.2 & 2 & 19 & 45 \\
\hline Carnation & Red & Black & 5.7 & 3 & 13 & 58 \\
\hline Clove & Brown & Yellow & 6.8 & 0 & 11 & 70 \\
\hline Whisky & Brown & Purple & 4.3 & 1 & 7 & 45 \\
\hline Butter & Yellow & Green & 4.0 & 0 & 6 & 45 \\
\hline Pineapple & Yellow & White & 4.9 & 2 & 3 & 56 \\
\hline Heptyl Acetate & Red & Gray & 4.0 & 3 & 2 & 45 \\
\hline Onion & White & Brown & 3.6 & 0 & 1 & 40 \\
\hline
\end{tabular}

Note-Odorant list order is decreasing initial recognition rates. Familiarity ratings are based on mean judgments. "ToN" indicates the number of TON states observed for the odorant. The sum of correct and incorrect initial identification rates is less than 1 by the rate of nonresponses. Identification rates are given in percentages. 
marshmallow candy shaped like peanuts." There is a commonly available candy that has amyl acetate as a component of the flavor; therefore, this response was scored as correct. This example illustrates a precise object description for a secondary source of the odorant. Imprecise identifications, the near misses, were not scored as correct. An example is the response "perfume" to the odorant carnation. Even with the exception rule described here, scoring odor identification responses remains biased toward underestimating identification rates. Some persons probably do provide precise identifications that are idiosyncratic to the individual. The response "dark, musty closet" is doubtless a veridical label for the odorant cedar, since many older homes have an infrequently used cedar paneled closet for storage purposes. However, accurate assessment of such responses would require individual interviews with each observer after the response data were analyzed-a burdensome procedure.

\section{RESULTS}

\section{Identification Rates}

Figure 1 presents the rates of correct $(\mathrm{C})$, incorrect (I), and no (NR) responses observed on the initial identification attempt and on the second attempt without an intervening context cue or after a word or color cue. Initial correct responding was about onehalf the rates reported elsewhere. On the second identification attempt, without any intervening context cue, there was a general reduction in responding, which decreased correct and incorrect responses by similar amounts.

The overall significance of the types of interpolated context cues was evaluated for each type of response. The Friedman two-way analysis of variance for related groups was selected (Friedman, 1940). This test is based on ranks of the number of responses given after each cue type. Table 3 presents the results of this analysis. First attempts to identify the odors produced uniform rates among the five samples. The $\mathrm{C}, \mathrm{I}$, and NR rates on the second attempt to identify the odors varied according to the cue type that preceded the response. The strongest effects were manifested by correct identification rates. The weakest influence of context was on the incorrect identification rate.

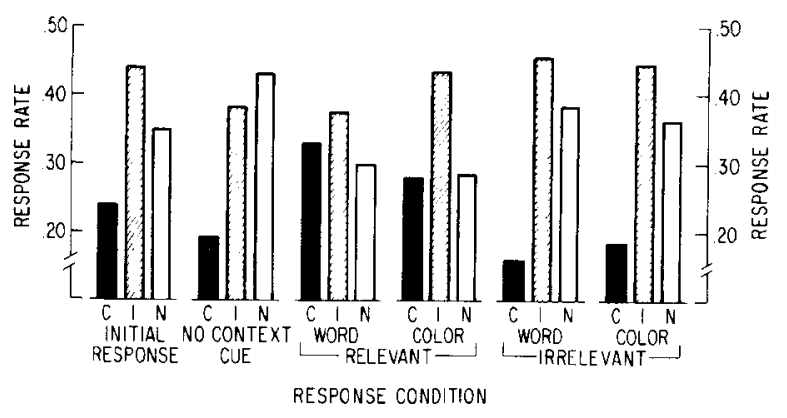

Figure 1. Rate of response for correct (C), incorrect (I), and no (NR) response types of identification responses on the initial attempt $(N=2,820)$ and on the five types of second attempt conditions ( $N=564$ in each condition).
Table 2

Odor Identification Rates

\begin{tabular}{|c|c|c|c|}
\hline \multirow{2}{*}{$\begin{array}{c}\text { First } \\
\text { Response }\end{array}$} & \multicolumn{3}{|c|}{ Second Response } \\
\hline & Correct & Incorrect & No Response \\
\hline & \multicolumn{3}{|c|}{ No Context Cue } \\
\hline Correct & 102 & 1 & 30 \\
\hline Incorrect & 1 & 186 & 61 \\
\hline \multirow[t]{2}{*}{ No Resporise } & 3 & 27 & 153 \\
\hline & \multicolumn{3}{|c|}{ Irrelevant Word Cue } \\
\hline Correct & 86 & 17 & 32 \\
\hline Incorrect & 3 & 179 & 63 \\
\hline \multirow[t]{2}{*}{ No Response } & 1 & 61 & 122 \\
\hline & \multicolumn{3}{|c|}{ Irrelevant Color Cue } \\
\hline Correct & 98 & 12 & 29 \\
\hline Incorrect & 4 & 187 & 61 \\
\hline \multirow[t]{2}{*}{ No Response } & 3 & 53 & 117 \\
\hline & \multicolumn{3}{|c|}{ Relevant Word Cue } \\
\hline Correct & 138 & 3 & 11 \\
\hline Incorrect & 36 & 174 & 37 \\
\hline \multirow[t]{2}{*}{ No Response } & 11 & 35 & 119 \\
\hline & \multicolumn{3}{|c|}{ Relevant Color Cue } \\
\hline Correct & 119 & 1 & 10 \\
\hline Incorrect & 18 & 192 & 45 \\
\hline No Response & 21 & 53 & 105 \\
\hline
\end{tabular}

The sign test was chosen to evaluate the relative differences between cue types. Wike and Church (1980) discuss this procedure. Since the $N$ for these paired comparisons ranged from 72 to 120 , the $\mathrm{z}$ approximation to the binomial distribution was used. Therefore, the stepped down sign test, as recommended by Wike and Church (1980), was not used.

The samples were ordered according to the mean ranks given in Table 3 for the second response. The resulting orders are shown in Table 4 . The underlines denote sets of conditions that are not different from each other but that do differ from other sets on the same line. These tests produced the following conclusions: (1) Relevant cues evoked more correct identifications than irrelevant or no-cue conditions. The relevant words were more potent than the colors. The irrelevant cues and the no-cue conditions did not differ among themselves on correct responses. (2) Irrelevant words were followed by more incorrect names than any other condition. The remaining conditions exhibited equal rates of incorrect responding. (3) The rate of NR (nonresponding) changed in the opposite direction relative to the rate of $\mathrm{C}$ (correct responding). Relevant cues encouraged responding, since this type of response had the lowest rate after relevant cues. Withholding a response was most likely to occur when the context cue did not intervene. Irrelevant cues reduced the tendency to withhold a response to a marginally significant degree.

The McNemar (1955) test of changes for two related samples was selected to evaluate the significance of the effects of each cue within each cue con- 
Table 3

Friedman Analysis of Variance for Related Samples Performed on Number of Each Response Type Within Cue Conditions Ranked Within Subjects

\begin{tabular}{|c|c|c|c|c|c|c|}
\hline \multirow{3}{*}{$\begin{array}{c}\text { Response } \\
\text { Type }\end{array}$} & \multicolumn{5}{|c|}{ Cue Type } & \multirow{3}{*}{$\begin{array}{c}\text { Friedman } \\
\text { Index }\end{array}$} \\
\hline & \multirow[b]{2}{*}{ No Cue } & \multicolumn{2}{|c|}{ Relevant } & \multicolumn{2}{|c|}{ Irrelevant } & \\
\hline & & Word & Color & Word & Color & \\
\hline & \multicolumn{6}{|c|}{ Initial } \\
\hline Correct & $2.92 *$ & 3.20 & 2.90 & 2.91 & 3.06 & $3.69 * *$ \\
\hline Incorrect & 2.96 & 2.97 & 3.11 & 2.96 & 2.99 & .93 \\
\hline \multirow[t]{2}{*}{ No Response } & 3.02 & 2.85 & 3.07 & 3.10 & 2.95 & 2.27 \\
\hline & \multicolumn{6}{|c|}{ Second } \\
\hline Correct & 2.71 & 3.59 & 3.34 & 2.55 & 2.84 & $50.9 \dagger \dagger$ \\
\hline Incorrect & 2.77 & 2.75 & 3.11 & 3.27 & 3.09 & $11.9+$ \\
\hline No Response & 3.43 & 2.70 & 2.54 & 3.21 & 3.12 & 31.1 \\
\hline
\end{tabular}

Note-The Friedman index is distributed as chi square with 4 degrees of freedom, 1 less than the number of samples being compared. *The mean of $n=141$ ranks for this cue and response condition. ${ }^{* *}$ Critical value for $p=.3$ is 4.88 . fCritical value for $p=.01$ is 11.67. tfCritical value for $p=.001$ is 18.46 .

dition. Three partitions were evaluated: (1) the tendency to change from making any response to no response or the reverse; (2) the tendency to change a successful response to an unsuccessful response (incorrect and no response combined) or the reverse; and (3) the tendency to change a correct response to an incorrect response or the reverse. These results will be considered in order of cue type.

Without an interpolated cue, the greatest tendency was to change from an initially wrong response to no response (91), instead of the reverse (30) $\left(\chi^{2}=29.75\right.$, $\mathrm{p}<.001)$. Having initially responded, the tendency to respond again in the opposite category was equal $\left(\alpha^{2}=.5, p>.4\right)$. Thus, if asked merely to try again, subjects who initially tried to identify the odor repeated their performance or stopped responding. Merely trying again did not improve the correct recognition rate.

Inserting a relevant color concept cue produced beneficial effects. After the relevant cue type, there

Table 4

Sign Test of Paired Comparisons Among the Cue Types on Second Identification Attempts

\begin{tabular}{|c|c|c|c|c|c|}
\hline \multirow{2}{*}{$\begin{array}{c}\begin{array}{c}\text { Response } \\
\text { Type }\end{array} \\
\text { Correct }\end{array}$} & \multicolumn{5}{|c|}{ Context Cue Type } \\
\hline & RW & RC & IRC & $\mathrm{NC}$ & IRW \\
\hline Incorrect & IRW & IRC & $\mathrm{RC}^{*}$ & RW & $\mathrm{NC*}$ \\
\hline No Response & $\mathrm{NC}$ & IRW & IRC & RW & RC \\
\hline
\end{tabular}

Note-The order of entry is decreasing summed ranks from Table 3. The underlines connect samples that are not significantly different for a $p<.05$ decision rule. $R W=$ relevant word; IRW $=$ irrelevant word; $R C=$ relevant color; IRC $=$ irrelevant color; $N C=$ no context cue. *Order reversed to aid conceptual clarity. was no tendency to change differentially from a responder to a nonresponder, which was true for no cues. The tendency to stop or to begin responding was the same after relevant words $\left(\chi^{2}=.01, \mathrm{p}>.8\right)$ and after relevant colors $\left(\chi^{2}=2.5, \mathrm{p}>.05\right)$. However, the tendency to change correctness was not equal. After the relevant words, significantly more persons changed from an incorrect to a correct response $\left(\chi^{2}=26.25, \mathrm{p}<.001\right)$. The relevant color cue also stimulated more changes from incorrect to correct responses $\left(\chi^{2}=13.47, \mathrm{p}<.001\right)$, but at a rate that was one-half the level produced by relevant words.

In contrast with the improvement produced by the relevant cues, the irrelevant cues were followed by diminished performance. When preceded by a response, the irrelevant words were followed by fewer responses $\left(\chi^{2}=6.52, p<.05\right)$. The irrelevant colors also produced fewer second responses after initial responses $\left(\chi^{2}=7.46, \mathrm{p}<.01\right)$. The changes produced by the irrelevant cues extended to reversals of initially correct responses for irrelevant words $\alpha^{2}=8.45$, $\mathrm{p}<.01)$. The effect with irrelevant colors was less and marginally nonsignificant $\left(\chi^{2}=3.06, .1<\mathrm{p}<.05\right)$. If overall failure is considered (incorrect responses and nonresponses combined), then both irrelevant words and colors were substantially influential in evoking increased identification failures for words $\left(\chi^{2}=36.53, \mathrm{p}<.001\right)$ and for colors $\left(\chi^{2}=22.69\right.$, $\mathrm{p}<.001)$. Thus, the irrelevant cues were incorporated and used by the subjects, which led to wrong responses.

To summarize the results: If no context cue is added, the subjects either repeated the initial guess or quit responding. If relevant cues intervened, the tendency was to furnish correct responses, which arose largely from no response on the first attempt. If irrelevant cues intervened, the initially correct responses and 
nonresponses were converted into wrong answers on the second attempt.

\section{Familiarity Effects}

Table 1 lists the mean familiarity rating assigned and the proportion of identification responses given to each odorant. The table is ordered according to the rate of correct identification. The mean identification rate was $.24(.16)$. The mean (SD) familiarity rating was $5.8(1.2)$. These two parameters correlated .73 .

Individual trials were sorted on the basis of the familiarity judgments into high, medium, and low levels of familiarity, where high represents categories 7,8 , and 9 . The rates of correct second responses, given a wrong first response, were determined within these intervals of familiarity for the five cue types. Figure 2 presents these results.

A correct recognition on the second response attempt, given a failure to give correct recognition on the first attempt, was taken to be the "tip of the nose" (TON) state followed by correct recall of the odor identification. Relevant context cues significantly increased TON conversion relative to the respective irrelevant cue condition. The irrelevant cues did not differ significantly from each other or from the no-context-cue conditions.

Note that subjects were not assigned randomly to TON or non-TON groups. Instead, the TON events

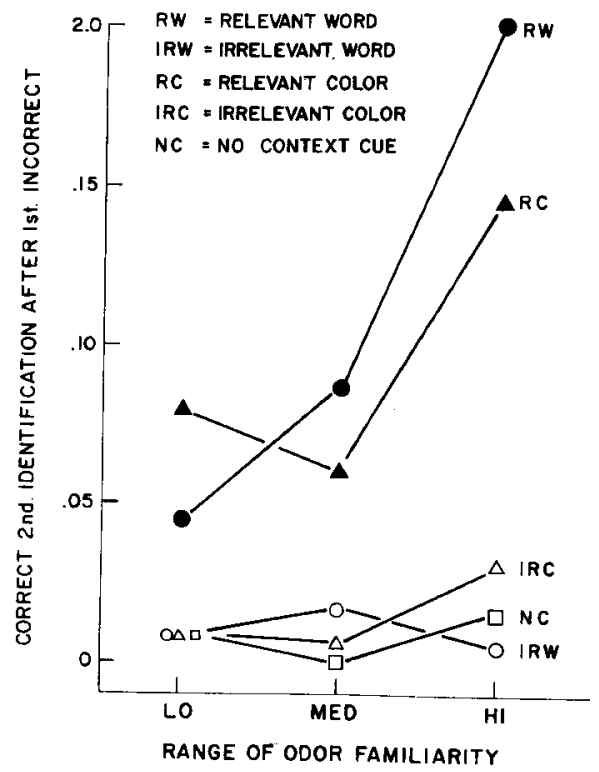

Figure 2. Identification rate on the second attempt, contingent upon the first attempt's being incorrect (including no response), related to the familiarity of the odor rated at the time of the first identification attempt. were a consequence of the experimental procedure. The comparisons given are essentially between persons. Among all subjects, 71 manifested a TON conversion, while 70 did not. Several methods of comparison between the TON and non-TON subjects were calculated. The mean familiarity judgment and the mean proportion of correct first identifications for each odor were abstracted for the two groups. Familiarity correlated .96 between the TON and nonTON groups, while proportion of correct responses correlated .93. The mean level of familiarity judgments was 5.7 and 5.8 for the TON and non-TON groups, respectively (not significantly different by $t$ test). The mean level of correct first identification was .23 and .26 for the TON and non-TON groups, respectively (not significantly different by $t$ test).

The familiarity and correct first identification rate were correlated within the TON and non-TON groups. The values correlated were averaged separately over subjects and then over stimuli. The subject mean familiarity and rate of correct first identification for subjects correlated .16 and .37 for the TON and nonTON groups, respectively. Averaged over odors, the mean familiarity and correct first identification rate correlated .72 and .72 for the TON and non-TON groups.

\section{DISCUSSION}

The general features of the results were as expected. The relevant cues encouraged increased responding and to a degree greater than the irrelevant cues. Moreover, the relevant cues evoked an increased rate of correct identifications. The presence of an irrelevant cue also increased response rates on the second attempt but reduced the rate of correct second responses. Thus, the irrelevant cue influenced the observers toward incorrect second guesses. The more cognitive "word" cues produced a more pronounced effect than did the "color" cues.

Subjects tend to repeat their first response on the second occasion. Three of four responses were of the consistent type, correct-correct, incorrectincorrect, or no response-no response. The no-cuetrial types had $78 \%$ consistency, the relevant words and colors had $74 \%$ and $76 \%$, respectively, and the irrelevant words and colors had $71 \%$ and $69 \%$, respectively. Thus, the influence of color concepts on identification can be manifested in only a small proportion of all trials.

When the context cue was effective in converting an initial wrong guess into a correct response, the odor was most likely to have been judged highly familiar. Thus, the context enhancement of identification is most potent when the observer is in a TON state. With regard to the primary question of this 
study, the results support the conclusion that disembodied odors experienced out of context are not identified to a substantially improved degree when a context cue is added.

The TON state appears to be a stochastic process that is distributed randomly among persons. There is a probability of .5 that a given person will exhibit one or more TON events in 20 opportunities. The TON observers did not differ from the non-TON persons in terms of identification rate or familiarity judgments. The only distinction detected between the TON and non-TON persons was the fact of emitting TON events.

In summary, if the context cue was relevant to the odors, the correct identification was facilitated, with word cues being more potent than the corresponding colors. In contrast, if the cues were irrelevant, both words and colors interfered with identification, with word cues again being more potent. When correct second responses, which follow initially incorrect first responses, are considered, the largest proportion were on trials in which a relevant context had been furnished. When each incorrect/correct trial was classified as to familiarity, the highly familiar odors were correctly identified four times more often relative to the medium and low familiarity odors.

These results are consistent with other reports on odor-naming behavior by Cain (1979), Davis (1975, 1977), Eich (1978), and Lawless and Engen (1977). Lawless and Engen studied the rate of occurrence of TON events and semantic atmosphere of the TON state.

A mixture of ecologically valid odorants (e.g., maple syrup) and odorous chemicals (e.g., coumarin), 48 in all, was presented by Lawless and Engen (1977) to 12 observers instructed to identify the odorants. These observers were instructed about the TON state and were asked to report when they judged themselves to be in a TON state. Of the $\mathbf{5 7 6}$ attempts to name an odor, $6.4 \%$ elicited TON states. In the present study, the uncued and irrelevant-cue-trial types elicited $.9 \%$ inferred TON events from 1,692 attempts, and the relevant cues elicited $7.6 \%$ TON events in 1,128 attempts. Even though Lawless and Engen (1977) used about $1 / 3$ "real" odor sources and alerted their subjects to the TON state, their observed rate does not seem remarkably different from that observed here. Moreover, their subjects reported in $50 \%$ to $75 \%$ of TON events that semantic responses, such as general odor class, origin as to place or object, and names of similar odors, were available while in the TON state.

Eich (1978) has examined the role of odor cues in human memory for verbal material, using a paradigm that was the reverse of that used in the present study. In Eich's study, the subjects were required to free-recall items from a 77 noun list after one study trial. These subjects performed an uncued recall, then an odor-cued recall, and then a semantic-cued recall, in which the odor of the two cued recall tasks was reversed for one-half of the subjects. The 77noun list included 12 target words that were odor names (e.g., coconut). The target words were cued with odors (e.g., the odor of coconut and the phrase "a flavor like macaroon" were used as cues in recall). Eich concluded that the odor as a cue was effective in enhancing verbal recall of the target words, but only after the odor was converted to an implicit semantic response. In this respect, the "critical determinant of effectiveness of a fragrance cue is the strength of the preexperimental associative bond the cue shares with the corresponding target word" (Eich, 1978).

Davis $(1975,1977)$ reported inferior acquisition of experimental verbal associates to odors that were highly distinctive and very familiar. These inferior performances were compared with acquisition with abstract and very unfamiliar graphic figures. Longterm retention of the verbal labels was equivalent for vision and olfaction, given equivalent initial performance levels. The "familiar" odor can "apparently activate preexisting cognitive mechanisms" (Davis, 1975), which facilitate verbal behavior. The implication is that this effect is at the semantic level of processing. Consequently, the failure to produce an odor name arises from deficient semantic mediation.

Cain (1979) observed a high rate of correct identification for $\mathbf{8 0}$ odors. The list was presented repeatedly, and the identification context was enriched with verbal cues. These cues were odor names furnished by Cain or the subjects themselves, and the cues were given immediately after the identification attempt. This procedure can be regarded as the highest degree of semantic enrichment of context. The correct identification rates exceeded $90 \%$ on the fourth trial in some conditions.

A common theme appears in the reports by Cain (1979), Davis (1975, 1977), Eich (1978), and Lawless and Engen (1977)-the dominant factor in verbal responses to odors is the preexisting associative bonds with specific verbal responses. Consequently, nonolfactory context cues cannot interact with olfactory information until all relevant stimuli are at the semantic response level of processing. Therefore, the TON state must arise when there is a failure of an odorant to evoke an implicit semantic response. At this level of processing, the semantic responses to the context cues do not have any primary semantic response arising from perception of the odor with which to interact.

In summary, the stimulus-impoverished odors used in laboratory studies are very difficult to identify due, primarily, to the olfactory information limitations. The contribution of nonolfactory con- 
text to identification can be positive, but the effect is very slight when compared with the enrichment of the olfactory stimulus (Desor \& Beauchamp, 1974).

\section{REFERENCES}

Catn, W. S. To know with the nose: Keys to odor identification. Science, 1979, 203, 467-470.

DAvis, R. G. Acquisition of verbal associations to olfactory stimuli of varying familiarity and to abstract visual stimuli. Journal of Experimental Psychology: Human Learning and Memory, 1975, 104, 134-142.

Davis, R. G. Acquisition and retention of verbal associations to olfactory and abstract visual stimuli of varying similarity. Journal of Experimental Psychology: Human Learning and Memory, 1977, 3, 37-51.

Davis, R. G. The microencapsulation of odorants as a method of stimulus control and delivery in studies of odor quality perception. Chemical Senses and Flavour, 1979, 4, 191-206.

Desor, J. A., \& Beauchamp, G. K. The human capacity to transmit olfactory information. Perception \& Psychophysics, $1974,16,551-556$.

EICH, J. E. Fragrances as cues for remembering words. Journal of Verbal Learning and Verbal Behavior, 1978, 17, 103-111.
Engen, T., \& Pfaffman, C. Absolute judgments of odor quality. Journal of Experimental Psychology, 1960, 59, 214-219.

ENGEN, T., \& Ross, B. Long-term memory of odors with and without verbal descriptions. Journal of Experimental Psychology, $1973,100,221-227$.

Friedman, M. A comparison of alternative tests of significance for the problem of $m$ rankings. Annals of Mathematical Statistics, 1940, 11, 86-92.

LAwLESS, H., \& ENGEN, T. Associations to odors: Interference, mnemonics, and verbal labeling. Journal of Experimental Psychology: Human Learning and Memory, 1977, 3, 52-59.

Matson, G. W. Microcapsules and process of making (U.S. Patent \#3 516 941). Washington, D.C: U.S. Patent Office, 1970.

McNemar, Q. Psychological statistics (2nd ed.). New York: Wiley, 1955.

Mozell, M., Smith, B., Smith, P., Sullivan, R., \& Swender, P. Nasal chemoreception in flavor identification. Archives of Otolaryngology, 1969, 90, 367-373.

TANYolac, N. Theories of odor and odor measurement. Istanbul: Robert College, 1968.

Wike, E. L., \& Church, J. D. Monte Carlo studies of Levy's "Nonparametric large sample pairwise comparisons." Psychological Bulletin, 1980, 88, 607-613.

(Manuscript received October 27, 1980; revision accepted for publication April 29, 1981.) 